those mentioned in this letter) by conducting randomized clinical trials and assessing protection against infection, rather than the ability to produce neutralizing antibodies because the former reflects the real-world effects of the vaccine more accurately. Governments and healthcare organizations must implement registry systems for breakthrough infections, to better understand the ongoing pandemic and outcomes of vaccination in the community. Finally, vaccines might be less effective against novel SARS-CoV-2 variants. Research institutes and vaccine producers should be prepared for such conditions and should develop new strategies to increase effectiveness against resistant lineages, such as introducing additional booster doses or reformulating the vaccines.

Acknowledgments.

Financial support. No financial support was provided relevant to this article.

Conflicts of interest. All authors report no conflicts of interest relevant to this article.

\section{References}

1. Share of COVID sequences that are the delta variant. Our World in Data website. https://ourworldindata.org/grapher/covid-cases-delta. Accessed July 20, 2021.

2. Share of people who received at least one dose of COVID-19 vaccine. Our World in Data website. https://ourworldindata.org/grapher/share-peoplevaccinated-covid?tab=table. Accessed July 20, 2021.
3. Daily new confirmed COVID-19 cases and deaths. Our World in Data website. https://ourworldindata.org/grapher/daily-covid-cases-deaths-7day-ra. Accessed July 20, 2021.

4. Hoffmann M, Hofmann-Winkler H, Krüger N, et al. SARS-CoV-2 variant B.1.617 is resistant to bamlanivimab and evades antibodies induced by infection and vaccination. Cell Rep 2021. doi: 10.1016/j.celrep.2021.109415.

5. Sheikh A, McMenamin J, Taylor R, Robertson C, Public Health Scotland and the EAVE II Collaborators. SARS-CoV-2 delta VOC in Scotland: demographics, risk of hospital admission, and vaccine effectiveness. Lancet 2021;397:2461-2462.

6. Lopez-Bernal J, Andrews N, Gower C, et al. Effectiveness of COVID-19 vaccines against the B.1.617.2 variant. medRxiv 2021. doi: 10.1101/2021.05.22. 21257658.

7. Nasreen $\mathrm{S}$, He S, Chung H, et al. Effectiveness of COVID-19 vaccines against variants of concern, Canada. medRxiv 2021. doi: 10.1101/2021.06.28. 21259420.

8. Independent Persian. ابتلاى كادر درمان به كرونا بِ از دريافت واكسن 2021 Available from: https://www.independentpersian.com/node/163041/. Accessed July 20, 2021.

9. Cheng H, Peng Z, Luo W, et al. Efficacy and safety of COVID-19 vaccines in phase III trials: a meta-analysis. Vaccines (Basel) 2021;9:582.

10. India's Bharat Biotech says vaccine is effective against delta variant. Wall Street Journal website. https://www.wsj.com/articles/indias-bharat-biotech-saysvaccine-is-effective-against-delta-variant-11625325879. Accessed July 20, 2021.

11. Russia's Sputnik V shot around $90 \%$ effective against delta variant, developers say. Reuters website. https://www.reuters.com/business/healthcarepharmaceuticals/russias-sputnik-v-vaccine-less-effective-against-deltavariant-tass-2021-06-29/. Accessed July 20, 2021.

\title{
SARS-CoV-2 vaccine breakthrough infections among healthcare workers in a large Belgian hospital network
}

\author{
Dieter Geysels $M D^{1}$ (1), Pierre Van Damme MD, prof. ${ }^{2}$, Walter Verstrepen PharmD, $\mathrm{PhD}^{1}$, Peggy Bruynseels $\mathrm{MD}^{1,3}$, \\ Bea Janssens $M D^{4}$, Patrick Smits $M D^{5}$ and Reinout Naesens MD ${ }^{1,3}$ \\ ${ }^{1}$ Department of Medical Microbiology, ZiekenhuisNetwerk Antwerpen, B-2020 Antwerp, Belgium, ${ }^{2}$ Centre for the Evaluation of Vaccination, Vaccine and Infectious \\ Disease Institute, University of Antwerp, B-2610 Wilrijk, Belgium, ${ }^{3}$ Department of Infection Prevention and Control, ZiekenhuisNetwerk Antwerpen, B-2020 \\ Antwerp, Belgium, ${ }^{4}$ IDEWE, External Service for Prevention and Protection at Work, B-2000 Antwerp, Belgium and ${ }^{5}$ Agency for Care and Health, Prevention \\ Division, Flanders, B-2018 Antwerp, Belgium
}

To the Editor-Since the first occurrence of SARS-CoV-2, different vaccines have been produced, tested, and approved in record time. However, the exact vaccination effect has yet to be determined in the ever-changing landscape of SARS-CoV-2 variants. ${ }^{1,2}$

\section{Breakthrough infections}

As of June 15, 2021, the overall vaccination rate in Belgium was $62.8 \%$ having received 1 dose (adult population), of whom $35.2 \%$ had fully completed their vaccination scheme. ${ }^{3}$ Belgian healthcare workers (HCWs) have a high exposure to COVID-19: the estimated percentage of confirmed Belgian COVID-19 cases is significantly higher among those working in healthcare facilities than among employees in other industries as well as the national average. ${ }^{4}$

Author for correspondence: Dr. Geysels Dieter, E-mail: dieter.geysels@zna.be

Cite this article: Geysels D, et al. (2022). SARS-CoV-2 vaccine breakthrough infections among healthcare workers in a large Belgian hospital network. Infection Control \& Hospital Epidemiology, 43: 1755-1757, https://doi.org/10.1017/ice.2021.326
Because HCWs were prioritized in the vaccination strategy, studying this population provided early data with which to analyze its effect. Vaccination in Belgium is not mandatory.

Vaccination of HCWs in ZNA started on January 18, 2021. ZNA is a 2,500-bed, public, multiple-site, hospital network in the Antwerp region. It comprises 3 acute-care hospitals, a children's hospital, and 5 chronic care facilities. Depending on their availability, 3 different vaccines were used: BNT162b2 (Comirnaty, BioNTech/Pfizer, Mainz, Germany), mRNA-1273 (COVID-19 Vaccine Moderna, Moderna, Cambridge, MA) and AZD1222 (Vaxzevria, Astra Zeneca, Cambridge, UK). The impact of vaccinations on the positive test ratio was evaluated from March 1 through April 30, 2021, a period with continuing and substantial viral circulation in the Belgian population. ${ }^{3}$ Tests were performed for contact tracing or COVID-like symptoms. Among 3,491 fully vaccinated ZNA HCWs, $9(0.3 \%)$ tested positive for SARS-CoV-2 (RT-PCR, Cobas 6800, Roche). After excluding 1 case, following CDC guidelines on persistent shedding, ${ }^{5} 22(1.0 \%)$ of 2,215 unvaccinated HCWs $(n=584)$ or partially vaccinated HCWs $(n=1,631)$ tested 
positive. Partially vaccinated was defined as having received only 1 dose or the second dose $<14$ days prior.

There were no significant differences between gender and age distribution for either group ( $P=0.6$ for gender; $P=0.3$ for age), with age ranging from 21 to 58 years. There were no known comorbidities or use of medication; among fully vaccinated HCWs, such factors might have explained a breakthrough infection.

Comparison of these proportions showed a significant difference between the 2 groups (odds ratio 3.9; 95\% confidence interval, $1.8-8.4 ; P<.001)$. Of the 9 HCWs who were fully vaccinated, 5 HCWs were vaccinated with the Comirnaty vaccine and 4 were vaccinated with the Moderna vaccine. Because the second dose of Vaxzevria could only be administered after 12 weeks, no HCWs were fully vaccinated with the latter.

Of the 31 HCWs, 26 were asymptomatic and discovered through contact tracing. In addition, 5 HCWs were tested because of symptoms compatible with COVID-19. Of these 5 HCWs, 2 were fully vaccinated (mRNA-1273). The cases of 18 HCWs who tested positive were all independent of the other cases. The other 13 cases were partially clustered in 6 groups working in the same ward: 5 groups of $2 \mathrm{HCWs}$ and 1 group with 3 linked cases.

\section{Viral loads}

Cycle threshold $(\mathrm{Ct})$ values were available for 7 of the 9 fully vaccinated HCWs and for 17 of the partially or unvaccinated HCWs, allowing viral load comparison (Fig. 1). Interestingly, fully vaccinated HCWs had relatively high viral loads: Ct values of 25.1 and 25.7 in 2 HCWs, respectively, corresponding to 4.6 and 4.4 $\log$ copies $/ \mathrm{mL}$. No significant differences in $\mathrm{Ct}$ values were observed between the 2 groups. The assumption that vaccination not only prevents severe disease and hospitalization but also diminishes the viral load once exposed ${ }^{6}$ was not substantiated by our data.

\section{Sequencing}

To gather more information about the influence of different strains, every breakthrough infection was genetically sequenced if the viral load was high enough to do so. Of the 7 fully vaccinated HCWs, 4 were eligible for whole-genome sequencing, and all 4 of these were infected with the B.1.1.7 strain. Of the unvaccinated and partially vaccinated HCWs, virus samples from 6 of these 17 were sequenced. Of these 6 SARS-COV-2-positive patients, 4 were infected with the B.1.1.7 strain as well, the other 2 had viral strains that originated from clade $20 \mathrm{~B}$, a variant first sequenced in Nigeria of unknown importance. During the investigation period, $~ 71 \%$ of the sequenced Belgian strains consisted of the B.1.1.7 strain $\left(>15,000\right.$ strains uploaded on Gisead). ${ }^{7}$

Our study has several limitations. One drawback of our investigation was the sample size. Although the study was performed in the largest hospital network in Belgium, our data are limited to Antwerp. We need larger, preferably international, studies with more statistical power to determine the true differences between these groups. Because this analysis was retrospective, we were unable to establish the baseline status of every employee before vaccination began. Invdividuals who shed the virus over an extended period (ie, "long-shedders") were included in our analysis, which may have distorted the true difference between the

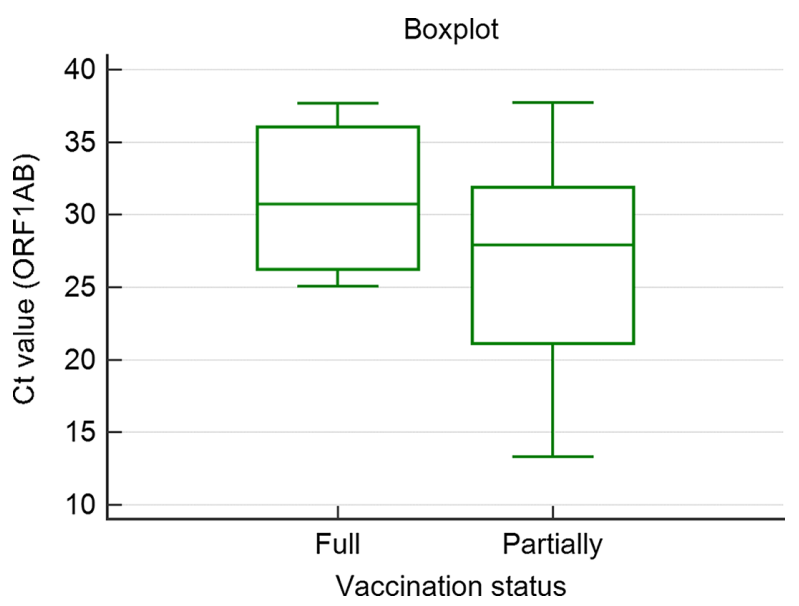

Fig. 1. Boxplot showing the distribution of cycle threshold (Ct) values of the ORF1AB gene. 'Partially' on the right shows the data of the nonvaccinated and incompletely vaccinated group, 'Full' on the left shows data for the fully vaccinated group.

groups. Information about the presence and titer values of antibodies to SARS-CoV-2 would have added value to our analysis as well.

In conclusion, vaccination led to a significant reduction in the incidence of SARS-CoV-2 infection rates in HCWs of an Antwerpbased multisite hospital. However, viral carriage was still present, and viral loads were not significantly lower than those of partially and unvaccinated HCWs. No information regarding an underlying immunodeficiency or relevant immunosuppressive medication was retained. The variants detected in the vaccinated HCWs reflected the current baseline epidemiology in the Antwerp region, where the dominant strain is B.1.1.7.

Not all patients have been vaccinated and substantial evidence suggests a lower efficacy in some immunosuppressed patients. ${ }^{8}$ Thus, we argue for maintaining strict contingency measures in the hospital setting.

\section{Acknowledgments.}

Financial support. No financial support was provided relevant to this article.

Conflicts of interest. Prof. Dr. Van Damme reported that the University of Antwerp receives research grants from GSK Biologicals, Pfizer, SANOFI, Merck, Themis, Osivax, J\&J and Abbott, The Bill \& Melinda Gates Foundation, PATH, the Flemish government, and the European Union outside the submitted work. All other authors report no conflicts of interest relevant to this article.

\section{References}

1. Hacisuleyman E, Hale C, Saito Y, et al. Vaccine breakthrough infections with SARS-CoV-2 variants. N Engl J Med 2021;384:2212-2218.

2. Gupta RK. Will SARS-CoV-2 variants of concern affect the promise of vaccines? Nat Rev Immunol 2021;21:340-341.

3. COVID-19- Epidemiologisch bulletin van 17 juni 2021. Sciensano website. www.sciensano.be. Published 2021. Accessed July 13, 2021.

4. COVID-19 incidentie in de actieve beroepsbevolking per sector. Sciensano website. https://covid-19.sciensano.be/nl/covid-19-epidemiologische-situatie. Published 2021. Accessed July 13, 2021.

5. Interim guidance on ending isolation and precautions for adults with COVID-19. Centers for Disease Control and Prevention website. https://www.cdc.gov/ coronavirus/2019-ncov/hcp/duration-isolation.html. Published 2021. Accessed July 13, 2021. 
6. Levine-Tiefenbrun M, Yelin I, Katz R, et al. Initial report of decreased SARSCoV-2 viral load after inoculation with the BNT162b2 vaccine. Nat Med 2021;27:790-792.

7. Baele G, Cuypers L, Maes P, et al. Genomic surveillance of SARS-CoV-2 in Belgium, situation update 4th of May 2021. National Reference
Laboratory UZ Leuven website. https://assets.uzleuven.be/files/2021-03/ genomic_surveillance_update_210211.pdf. Published 2021. Accessed July $13,2021$.

8. Sonani B, Aslam F, Goyal A, Patel J, Bansal P. COVID-19 vaccination in immunocompromised patients. Clin Rheumatol 2021;40:797-798.

\title{
Coronavirus disease 2019 (COVID-19) infection of healthcare personnel after complete COVID-19 vaccination
}

\author{
Rujittika Mungmunpuntipantip ${ }^{1}$ and Viroj Wiwanitkit ${ }^{2}$ \\ ${ }^{1}$ Private Academic Consultant, Bangkok, Thailand and ${ }^{2}$ Honorary professor, Dr DY Patil University, Pune, India
}

To the Editor-We would like to share our ideas on the recent article, "Healthcare-associated transmission of severe acute respiratory coronavirus virus 2 (SARS-CoV-2) among Thai healthcare personnel who receive 2 doses of a coronavirus disease 2019 (COVID-19) vaccine: A call for considering a booster dose." Apisarnthanarak et al proposed that "HCP were at extremely high risk for healthcare-associated SARS-CoV-2 transmission despite receiving 2 doses of COVID-19 ... suboptimal immune response following CoronaVac, particularly after day $60 .{ }^{\prime \prime}$ The COVID-19 infection of healthcare personnel might occur despite complete vaccination. There are many possible causes of vaccine failure. Apisarnthanarak et al proposed the poor immunogenicity of the vaccine, which is an important factor. ${ }^{1}$ Different kinds of vaccines have different immunogenicity mechanisms and different levels of effectiveness. In addition, the temperature control of vaccine storage and distribution is a forgotten issue. Thailand is a tropical country where local temperatures are very hot, and vaccine might not be well kept or stored at the recommended temperature. In addition, the vaccination technique can also cause vaccine failure; a recent report from Thailand detailed complications of vaccination due to poor administration techniques. ${ }^{2}$ Finally, good environmental control in clinical wards and the self-prevention practice (eg, face mask compliance) among employees after vaccination are also very important considerations. The belief or misinformation that vaccination absolutely prevents severe infection might lead to careless infection prevention behavior.

\section{Acknowledgments.}

Financial support. No financial support was provided relevant to this article.

Conflicts of interest. All authors report no conflicts of interest relevant to this article.

\section{References}

1. Apisarnthanarak A, Nantapisal S, Pienthong T, Apisarnthanarak P, Weber DJ. Healthcare-associated transmission of severe acute respiratory coronavirus virus 2 (SARS-CoV-2) among Thai healthcare personnel who receive 2 doses of a coronavirus disease 2019 (COVID-19) vaccine: a call for considering a booster dose. Infect Control Hosp Epidemiol 2021. doi: 10.1017/ice.2021.365.

2. Chuaychoosakoon C, Parinyakhup W, Tanutit P, Maliwankul K, Klabklay P. Shoulder injury related to Sinovac COVID-19 vaccine: a case report. Ann Med Surg (Lond) 2021;68:102622. 